\title{
Global identification of full-length cassava lncRNAs unveils the role of CRIR1 in cold stress response
}

\author{
shuxia $\mathrm{li}^{1}$, Zhihao Cheng ${ }^{1}$, Shiman Dong ${ }^{1}$, Zhibo Li $^{1}$, Liangping Zou ${ }^{1}$, Pingjuan Zhao ${ }^{1}$, Xin \\ $\mathrm{Guo}^{1}$, Yan $\mathrm{Bao}^{2}$, Wenquan $\mathrm{Wang}^{3}$, and Ming Peng ${ }^{4}$ \\ ${ }^{1}$ Chinese Academy of Tropical Agricultural Sciences \\ ${ }^{2}$ Shanghai Jiao Tong University \\ ${ }^{3}$ Hainan University \\ ${ }^{4}$ Institute of Tropical Bioscience and Biotechnology, Chinese Academy of Tropical \\ Agricultural Sciences
}

October 15, 2021

\begin{abstract}
Long non-coding RNAs (lncRNAs) have been considered to be important regulators of gene expression in a range of biological processes in plants. A large number of lncRNAs have been identified in plants. However, most of their biological functions still remain to be determined. Here, we identified total 3004 lncRNAs in cassava under normal or cold-treated conditions from Isoseq data. We further characterized a lincRNA, CRIR1, as a novel positive regulator of the plant response to cold stress. CRIR1 can be significantly induced by cold treatment. Overexpression of CRIR 1 in cassava enhanced the cold tolerance of transgenic plants. Transcriptome analysis demonstrated that CRIR1 regulates a range of cold stress-related genes in a CBF-independent pathway. We further found that CRIR1 RNA can interact with MeCSP5, a homolog of the cold shock protein that acts as RNA chaperones, indicating that CRIR1 may recruit MeCSP5 to improve the translation efficiency of mRNA. In summary, our study greatly extends the repertoire of lncRNAs in plants as well as its responding to cold stress. Moreover, it reveals a sophisticated mechanism by which CRIR1 regulates plant cold stress response by modulating the expression of stress-responsive genes and increasing the translational yield.
\end{abstract}

\section{INTRODUCTION}

Cassava (Manihot esculenta Crantz) is a starchy root crop that are widely cultivated throughout the tropics and the subtropics. Over the last few decades, the demand for cassava has changed rapidly from a direct food crop to an industrial crop, mainly as human food, animal feed, biofuels, and other bio-based materials (Malik, Kongsil, Nguyen, Ou, Sholihin, Srean, Sheela, Becerra Lopez-Lavalle, Utsumi, Lu, Kittipadakul, Nguyen, Ceballos, Nguyen, Selvaraj Gomez, Aiemnaka, Labarta, Chen, Amawan, Sok, Youabee, Seki, Tokunaga, Wang, Li, Nguyen, Nguyen, Ham \& Ishitani, 2020). However, as a tropical and subtropical crop plant, cassava is very sensitive to cold stress, which drastically impairs its seedling growth, photosynthesis, and significantly limits the root weight (E1-Sharkawy, 2004). Severe cold stress is particularly damaging to its leaves and apical meristems, leading to stunted growth and even death (An, Yang \& Zhang, 2012). Therefore, cold stress is a major limiting factor for cassava productivity and spatial distribution.

In order to cope with cold stress, plants have developed a complex network of pathways to sense and respond to rapid fluctuations in temperature (Zhu, 2016). During the past two decades, the underlying mechanisms of the molecular and physiological changes that enable an adaptive response to cold stress in plants have been extensively explored. Studies in Arabidopsis lead to the identification of a set of coldrelated transcription factors, such as C-REPEAT-BINDING FACTORS (CBFs), MYBs, NACs, that can 
regulate downstream genes, leading to biochemical and physiological adjustments at the cellular and plant levels under cold condition (Ding, Shi \& Yang, 2020, Nuruzzaman, Sharoni \& Kikuchi, 2013, Shi, Ding \& Yang, 2018, Zhao, Zhang, Xie, Si, Li \& Zhu, 2016). The CBF proteins play a central role in plant cold acclimation by specifically binding to the CBF responsive element (CRT) motifs presenting in the promoter regions of COLD-RESPONSIVE (COR) genes, such as RD29a and COR15a, to activate their transcription (Shi et al., 2018). In cassava, the $C B F$ genes are rapidly and transiently upregulated by cold stress (An et al., 2012). Overexpression of certain MeCBF3 can enhance the cold tolerance of cassava (An, Ma, Wang, Yang, Zhou \& Zhang, 2017). In addition to basic transcriptional regulation, post-transcriptional regulation, such as pre-mRNA splicing, mRNA transport, and mRNA stability, also influence the cold stress response regulatory network in plants (Chinnusamy, Gong \& Zhu, 2008, Lee, Kapoor, Zhu \& Zhu, 2006).

Similar to proteins, the functions of many cellular RNAs directly rely both on their nucleotide sequences and their spatial structures (Rajkowitsch, Chen, Stampfl, Semrad, Waldsich, Mayer, Jantsch, Konrat, Blasi \& Schroeder, 2007). Dynamic, environment-mediated structural changes of RNA molecules can modulate their transcription, maturation, and translation efficiency. Cold stress causes overstabilization of incorrectly folded RNA structures, while RNA chaperones can resolve these structures, maintaing the normal function of the RNAs (Melencion, Chi, Pham, Paeng, Wi, Lee, Ryu, Koo \& Lee, 2017). COLD SHOCK PROTEINS ( $C S P s$ ), characterized by the presence of cold shock domains, are broadly found as nucleic acid chaperones in bacteria, animals, and plants (Sasaki \& Imai, 2011). In bacteria and humans, the biological activities of CSPs range from the regulation of transcription and splicing, to the translation of mRNAs (Lindquist \& Mertens, 2018). In plants, CSPs are involved in various biological processes to promote normal growth and stress responses. The first functionally characterized plant CSP gene was wheat WCSP1 with cold-specific inductions, and the corresponding protein melted double-stranded nucleic acids, fulfilling various cellular functions (Karlson, Nakaminami, Toyomasu \& Imai, 2002, Nakaminami, Karlson \& Imai, 2006). Arabidopsis has four CSP proteins (AtCSP1-AtCSP4) that play distinct roles in plant development and stress responses. For example, AtCSP3 can be induced by cold stress, while overexpression of AtCSP3 conferred enhanced cold tolerance without obvious developmental defects (Kim, Sasaki \& Imai, 2009). However, overexpression of AtCSP1 or AtCSP2 did not enhance cold tolerance in Arabidopsis, but complemented the cold-sensitive phenotypeof grp 7 mutant, and affected seed germination under salt stress conditions (Kim, Park, Kwak, Kim, Kim, Song, Jang, Jung \& Kang, 2007, Park, Kwak, Oh, Kim \& Kang, 2009, Yang \& Karlson, 2013). Therefore, activation of CSP proteins is essential and may enhance the ability of the plants to cope with cold stress.

Previous studies on stress gene regulation mainly focused on protein-coding genes. In recent years, long non-coding RNAs (lncRNAs) have emerged as essential regulators of genomic and phenotypic diversities (Waititu, Zhang, Liu \& Wang, 2020). LncRNAs are longer than 200 nt and usually have low protein-coding potential. In plants, most lncRNAs are produced by RNA polymerase II (Pol II), whereas some lncRNAs are transcribed by RNA Pol III, IV, and V (Wang, Meng, Dobrovolskaya, Orlov \& Chen, 2017). LncRNAs are involved in various regulatory events by acting as precursors of small RNAs, such as miRNAs and siRNAs, or as miRNA target mimics. They are also reported to be involved in gene expression regulation via multiple ways, including transcriptional activation/repression, RNA alternative splicing, and chromatin modification (Csorba, Questa, Sun \& Dean, 2014, Peschansky \& Wahlestedt, 2014, Rigo, Bazin, Romero-Barrios, Moison, Lucero, Christ, Benhamed, Blein, Huguet, Charon, Crespi \& Ariel, 2020, Zhao, Li, Lian, Gu, Li \& Qi, 2018). With RNA sequencing technologies and experimental approaches, a great number of lncRNAs have been identified and confirmed to play key roles in plant response and adaptation to abiotic stresses. For instance, drought and salt stress tolerance of plants can be enhanced by transgenic Arabidopsis overexpressing a nucleus-localized lncRNA (Qin, Zhao, Cui, Albesher \& Xiong, 2017). A natural antisense transcript of maize $Z m N A C 48$ were reported to interact with sense mRNAs during the drought stress response, resulting in the formation of nat-siRNA, which could inhibit the expression of $Z m N A C 48$ to affect the stomatal closure of maize (Mao, Xu, Wang, Li, Tang, Liu, Feng, Wu, Li, Xie \& Lu, 2021). In poplar, two heat-responsive lncRNAs have been shown to modulate target gene expression via RNA interference and act as RNA scaffolds to enhance heat tolerance (Song, Chen, Liu, Bu \& Zhang, 2020). 
In cassava, several previous studies have identified a great number of stress-responsive lncRNAs, and their regulatory networks have been predicted (Ding, Tie, Fu, Yan, Liu, Yan, Li, Wu, Zhang \& Hu, 2019, Li, Yu, Lei, Cheng, Zhao, He, Wang \& Peng, 2017, Xiao, Shang, Cao, Xie, Zeng, Lu, Chen \& Yan, 2019). However, only the short-read RNA sequencing platform was used in these studies, which barely provided full-length lncRNAs based on the bioinformatic algorithms, and the number of full-length lncRNAs transcribed from Pol II was unclear. The experimental validation of their functions remains challenging and has not yet been reported. Therefore, it is necessary to construct a complete resource of full-length lncRNAs and elucidate the mechanisms of their actions for the cassava lncRNAs, which may provide helpful information for crop breeding. In our previous study, we reported a large-scale study of alternative splicing dynamics under abiotic stress conditions in cassava by analyzing single-molecule long-read isoform sequencing (Isoseq) data (Li, Yu, Cheng, Zeng, Li, Zhang \& Peng, 2020). Here, we performed an integrative analysis of long-read and short-read transcriptome sequencing data from the cassava shoot apex under cold and normal conditions and identified a total of 3004 full-length lncRNAs. These data could add into the annotation of thousands of cassava lncRNA genes and serve as reference sequences for further in-depth gene function studies. Subsequently, we identified and characterized the COLD-RESPONSIVE lincRNA 1 (CRIR1). Gain-of-function analysis revealed that CRIR1 overexpression transgenic cassava seedlings conferred tolerance to cold stress. RNA sequencing (RNA-seq) analysis indicated that a range of coldresponsive genes was induced in CRIR1 -overexpressing transgenic lines. Furthermore, we found that CRIR1 interacted directly with the cold shock protein MeCSP5 in cassava cells, and increased its translational yield to cope with cold stress. In conclusion, these results strongly suggest that CRIR 1 has a pivotal role in manipulating cold-stress tolerance in cassava, possibaly though the regulation of MeCSP5.

\section{MATERIALS AND METHODS}

\subsection{Plant materials, growth conditions, and cold treatment}

Cassava cultivar TMS60444 was used in this study. The uniform stem cuttings were grown on Murashige and Skoog (MS) medium containing $3.0 \mathrm{~g} / \mathrm{L}$ Gelrite, $20 \mathrm{~g} / \mathrm{L}$ sucrose (Fisher), and $2 \mu \mathrm{mol} / \mathrm{L} \mathrm{CuSO}_{4}$ in a growth room at $26^{\circ} \mathrm{C}$ under $16 \mathrm{~h}$ light $/ 8 \mathrm{~h}$ dark. For cold treatment, 4-week-old seedlings were transplanted into pots and grown in a greenhouse $\left(16 \mathrm{~h}\right.$ days, $\left.26 \pm 2{ }^{\circ} \mathrm{C}\right)$. After two weeks, seedlings with a uniform growth status were transferred to a chamber for cold treatment at $4^{\circ} \mathrm{C}$ under light, followed by one week of recovery at $26^{\circ} \mathrm{C}$. More than 5 plants were treated in each treatment.

\subsection{Plasmid construction and cassava transformation}

The cDNA sequences of the sense and antisense CRIR1 were cloned by PCR using primers covering the full length of transcripts. The PCR fragment was sequenced and inserted into the binary vector pCAMBIA1301 under the control of the Cauliflower Mosaic Virus (CaMV) 35S promoter to generate the binary vector pC35S ::CRIR1 and pC35S ::anti-CRIR1, respectively. The plasmid was mobilized into Agrobacterium tumefaciens LBA4404 for cassava transformation using a friable embryogenic callus of cultivar TMS60444. Cassava embryogenic callus induction, Agrobacterium -mediated genetic transformation, and hygromycinresistant calli regeneration were performed by the methods described previously (Zhang, Potrykus \& PuontiKaerlas, 2000).

\subsection{Southern blot analysis of transgenic plants of transgenic plants}

The southern blot assay was performed as described previously (An et al., 2017). Briefly, mature leaves of wild-type (WT) and transgenic plant were harvested for genomic DNA isolation. Approximately $20 \mu \mathrm{g}$ of total DNA was digested with EcoRI overnight, followed by separating on a $0.8 \%(\mathrm{w} / \mathrm{v})$ agarose gel and subsequently transferred to a positively charged nylon membrane (Roche, Mannheim, Germany). The blot was hybridized with the digoxigenin (DIG)-labeled hygromycin phosphotransferase (HPT) gene probe, which was generated by using the PCR DIG Probe Synthesis Kit (Roche, Mannheim, Germany). According to the manufacturer's instructions, hybridization, membrane washing, and detection were performed using the DIG-High Prime DNA Labeling and Detection Starter Kit II (Roche, Mannheim, Germany). 


\subsection{Physiological analyses of cold-treated cassava plants}

Malondialdehyde (MDA) content in cassava leaves was determined by using Lipid oxidation detection kit (Beyotime) according to the manufacturer's instructions. MDA was extracted with chilled thiobarbituric acid reagent, and then the MDA contents were calculated by subtracting the absorbance at 450 and 600 $\mathrm{nm}$ from that at $532 \mathrm{~nm}$. Proline concentrations were measured by using Proline Assay Kit (Bioss, Beijing) according to the manufacturer's instructions. Total protein extraction, purification, and determination were performed by Sanshu Biotechnology Co., Ltd (Jiangsu, China). Each treatment had three replications.

\subsection{RNA isolation and quantitative real-time PCR}

The young leaves and shoot buds were harvested after $24 \mathrm{~h}$ treatment at $4^{\circ} \mathrm{C}$, then frozen in liquid nitrogen for RNA extraction. Nonstressed seedlings were harvested as controls $(0 \mathrm{~h})$. More than 6 plants were harvested and pooled in each treatment. Total RNA was extracted using the Plant RNA Kit (Omega BioTek). First-strand cDNA was synthesized using the PrimeScript RT Reagent Kit (Takara, Otsu, Japan). A quantitative real-time PCR (qRT-PCR) was conducted using SYBR (r) Premix Ex Taq (Takara) and the StepOnePlus Real-Time PCR Detection System (Bio-Rad, CA, USA). MeACTIN was used as a reference gene for normalizing transcript levels. Details regarding the primers used in this study are provided in Table S3.

\subsection{Iso-Seq and ssRNA-Seq data processing and lncRNA identification}

The RNA preparation, library construction, and sequencing for Iso-Seq (Li et al., 2020) and ssRNA-Seq (Li et al., 2017) were described previously. All sequencing data were deposited with NCBI under the BioProject ID PRJNA198574 and PRJNA377165. For Iso-Seq, total RNA was extracted using TRIzol reagent (Life technologies) and enriched by Oligo (dT) magnetic beads. The enriched mRNA was reverse transcribed into cDNA using Clontech SMARTer PCR cDNA Synthesis Kit. A total of two libraries (Normal and Cold) were constructed and sequenced on the Pacific Biosciences (PacBio) Sequel II platform by Gene Denovo Biotechnology Co., Ltd. (Guangzhou, China). The raw reads were classified and clustered into transcript consensus using SMRT Link v5.0.1 pipeline (Gordon, Tseng, Salamov, Zhang, Meng, Zhao, Kang, Underwood, Grigoriev, Figueroa, Schilling, Chen \& Wang, 2015) supported by PacBio and then mapped to reference genome using minimap2 ( $\mathrm{Li}, 2018$ ). Long non-coding RNA identification was performed according to the pipeline described previously ( $\mathrm{Li}$ et al., 2017). The intersection of both non-protein-coding potential results and non-protein annotation results were chosen as lncRNA candidates. For ssRNA-Seq data processing, clean reads from two samples (Normal and Cold, 3 replicates per sample) were mapped to the full-length lncRNA isoforms and cassava reference genome by HISAT (Kim, Langmead \& Salzberg, 2015). The counts of each lncRNA were quantified by RSEM (Li \& Dewey, 2011), and the quantitative estimation of each transcript was achieved using fragments per kilobase of exon model per million mapped reads (FPKM). Differential expressed lncRNAs were analyzed by the DESeq2 package (Love, Huber \& Anders, 2014). Significant changes were determined using $|\log 2 \mathrm{FC}|>1$ and q-value (false discovery rate, FDR $<5 \%$ ) from multiple-testing adjustment as cut-off.

\subsection{Illumina RNA sequencing and data analysis}

Young leaves and shoot tips from four-week-old WT and CRIR1 OE lines grown under normal and coldtreated $(4 \mathrm{degC}, 24 \mathrm{~h})$ conditions were harvested. Two biological replicates consisting of six independent plants for each sample were conducted. The total RNA isolation, whole transcriptome libraries preparation, and deep sequencing were performed by Gene Denovo Biotechnology Co., Ltd. (Guangzhou, China). Total RNA from each sample was extracted using Trizol reagent (Life Technologies). According to the manufacturer's instructions, whole transcriptome libraries were constructed using Next(r) Ultra RNA Library Prep Kit (New England Biolabs). The libraries were sequenced initially on an Illumina NovaSeq ${ }^{\mathrm{TM}} 6000$ instrument that generated paired-end reads of 150 nucleotides. After removing the adapters and low-quality bases, clean reads were aligned to the cassava genome assembly with HISAT (Kim et al., 2015), then transcript construction using Stringtie (Pertea, Pertea, Antonescu, Chang, Mendell \& Salzberg, 2015). The FPKM value was calculated for each unigene using RSEM (Li \& Dewey, 2011). The differentially expressed genes 
(DEGs) were identified by using DESeq2 (Love et al., 2014), with FDR $<0.05$. Gene Ontology (GO) enrichment analyses were conducted using AgriGO (Tian, Liu, Yan, You, Yi, Du, Xu \& Su, 2017). The RNA-seq data have been deposited to the National Center for Biotechnology Information (NCBI) under the accession number PRJNA522309.

\subsection{Fluorescence in situ hybridization (FISH) analysis}

The samples for ISH were prepared as described previously (Yang, Schuster, Prunet, Dong, Landrein, Wightman \& Meyerowitz, 2019). Full expanded young leaves from three-week-old cassava seedlings were fixed in $4 \%$ paraformaldehyde for $20 \mathrm{mins}$, and the leaves were then dehydrated and embedded in paraffin. Subsequently, the tissue blocks were cut into $7-\mu \mathrm{m}$ sections, processed by dewaxing, rehydration, and dehydration. FISH analysis was performed using an RNA-FISH kit (BersinBio, Guangzhou, China), a CRIR1 probe labeled with $\mathrm{Cy} 3$ was designed and constructed by BersinBio. The dehydrated sections were pre-hybridized in a $37^{\circ} \mathrm{C}$ incubator for $0.5 \mathrm{~h}$. The hybridization solution with Cy3-labeledCRIR1 probes was added, and the slide was allowed to hybridize overnight at $4 \mathrm{degC}$. The sections were washed once in SSC (2x) for 10 mins, twice in SSC $(1 \mathrm{x})$ for 5 mins each, and once in SSC $(0.5 \mathrm{x})$ for 10 mins. Finally, the samples were stained with (DAPI) dye solution, incubated in the dark for 8 mins, washed with PBS, and photographed with a laser confocal microscope (Olympus FluoView FV1100).

\subsection{TrifC Assay}

In the case of TriFC assay, the full-length coding sequences for MeCSP5 and MSCP were cloned into pSAT4nEYFP-C1 and pSAT4-cEYFP-C1 vectors, respectively. The sequence of 6xMS2 was fused with CRIR1 or control lncRNA under the control of the 35S CaMV promoter in the pCAMBIA1301 vector. For the TriFC assay in rice protoplasts, transient transfection of rice protoplast cultures were performed according to the protocol described previously (Li, Xiang, Yang, Chen \& Zhang, 2015). Fluorescence was detected using confocal laser scanning microscope (Olympus FluoView FV1100) 16-20 h after transfection.

\subsection{Histological Analysis}

For transmission electron microscopy, cassava leaves were fixed in $2.5 \%$ glutaraldehyde then transferred to osmium tetraoxide. The fixed samples were dehydrated in an ethanol gradient then transferred to propylene oxide solution, after which they were slowly polymerized in acrylic resin (London Resin Company) for at least $48 \mathrm{~h}$. Thin sections $(70 \mathrm{~nm}$ ) were obtained using a diamond knife microtome (Ultracut E; Reichert Technologies). The sections were placed on 100-mesh copper grids, stained with uranyl acetate for 30 mins, and then stained with lead citrate for 15 mins. The sections were observed using a Hitachi HT7700 transmission electron microscope (Japan).

\subsection{Biotin RNA Pull-Down Assay}

RNA pull-down assay was performed as previously described (Hu, Jin, Xu, Wang, Thorne, Zhang \& Wu, 2018). Biotin-labeled CRIR1 or Anti-CRIR1 RNAs were transcribed in vitro using the Biotin RNA Labeling Mix (Roche) and T7 RNA polymerase (Roche), treated with RNase-free DNase I (Invitrogen), and purified with RNeasy Mini Kit (Qiagen). Total protein was extracted from 3-week-old cassava seedlings using Total Protein Extraction Kit for plant tissues (Invent Biotechnologies, Inc) and pre-cleared against streptavidin magnetic beads (Invitrogen). Biotin-labeled CRIR1 RNAs adsorbed to streptavidin magnetic beads were then incubated with pre-cleared protein extract in the presence of complete protease inhibitors cocktail, phosphatase inhibitor cocktail, and RNase inhibitors, at $4 \operatorname{degC}$ for $12 \mathrm{~h}$, followed by washing five times in wash buffer $(100 \mathrm{mM} \mathrm{KCl}, 50 \mathrm{mM}$ Tris $(\mathrm{pH} 7.4), 0.5 \mathrm{mM}$ dithiothreitol, $0.5 \%$ NP-40, 1mM DTT and RNase inhibitors) and elution in 5xSDS sample loading buffer. Eluted proteins were separated by SDS-PAGE and were then subjected to mass spectrometric analysis.

\subsection{Liquid Chromatography-Mass Spectrometry (LC-MS) analysis}

Proteins purified from RNA pull-down assay were analyzed using LC-MS by the Shanghai Applied Protein Technology Co., Ltd (Shanghai, China). Two libraries (CRIR1 and Anti-CRIR1), which contained two 
replicates for each library, were prepared in this study. Dry pellets of samples were solubilized in trypsin buffer (Promega) for digestion into small peptides. The digested peptides were desalted and solubilized in $0.1 \%$ formic acid buffer. The peptide mixture was loaded onto a reverse-phase trap column (Thermo Scientific Acclaim PepMap100) connected to the C18 reversed-phase analytical column (Thermo Scientific Easy Column, $10 \mathrm{~cm}$ long, $75 \mu \mathrm{m}$ inner diameter, $3 \mu \mathrm{m}$ resin) in buffer A (0.1\% Formic acid) and separated with a linear gradient of buffer B ( $84 \%$ acetonitrile and $0.1 \%$ Formic acid). LC-MS/MS analysis was performed on a Q Exactive mass spectrometer (Thermo Scientific) coupled to Easy nLC (Proxeon Biosystems, now Thermo Fisher Scientific). MS data were acquired using a data-dependent top10 method dynamically choosing the most abundant precursor ions from the survey scan $(300-1800 \mathrm{~m} / \mathrm{z}$ ) for HCD fragmentation. Data were analyzed for each sample with MaxQuant software (Cox \& Mann, 2008).

\subsection{Electrophoretic mobility shift assay (EMSA)}

The electrophoretic mobility shift assay was conducted using an RNA EMSA Kit (BersinBio, Guangzhou, China) according to the manufacturer's instructions. Briefly, the full-length CDS of MeCSP5 was amplified by PCR and then cloned into the pCzn1-His to express the MeCSP5 protein in Escherichia coli DE3 strain. The recombinant MeCSP5 protein was purified using the Ni affinity chromatography. EMSA was performed in a reaction mixture containing MeCSP5 proteins and biotin-labeled CRIR1 RNA probes. Negative control assays were performed by adding 100-fold excesses of unlabeled RNA probes to the reaction mixture. After electrophoresis and incubation, signals were recorded and analyzed.

\subsection{Accession Numbers}

All sequence data from this article can be found under accession numbers: MeCSP5 (Manes.18G074700), MeNAC(Manes.01G093200), MeNFYA (Manes.09G025200), MeGOLS(Manes.03G208800), MeASR (Manes.18G093100), MeYAB2(Manes.04G064600), MeIAA18 (Manes.04G134400), MeABP19(Manes.04G144900), MeGRF5 (Manes.04G144700), MeCBF3(Manes.08G096900), MeCBF4 (Manes.07G135300), MeCOR(Manes.18G037600), MeICE1 (Manes.04G092400).

\section{RESULTS}

\subsection{Overview of cassava lncRNAs detected by long-read and short-read transcriptome sequenc- ing}

In this study, through the combined analysis of Iso-Seq and ssRNA-seq data, a total of 70146 unique transcripts were obtained, including $95.7 \%$ predicted protein-coding and $4.3 \%$ long non-coding RNA. The number of predicted lncRNAs based on Iso-seq data is shown in Figure 1a. A total of 3004 high-confidence full-length lncRNAs were identified with an average length of 1424 bp. To study these lncRNAs in detail, we classified them into 5 different categories based on their genomic distribution and potential functions. Among these lncRNAs, a large proportion (44.6\%) represented sense lncRNAs, $19.5 \%$ of them were produced from the antisense strand of protein-coding genes, while $19.2 \%$ of them appeared to be intergenic non-coding RNAs. LikeArabidopsis lncRNAs (Liu, Jung, Xu, Wang, Deng, Bernad, Arenas-Huertero \& Chua, 2012), only a few cassava lncRNAs (11 of 3004 lncRNAs) acted as precursor transcripts of miRNAs or siRNAs after aligning miRNA sequences from miRbase to our lncRNAs collection (Table S1). We then estimated the expression level of each transcript using FPKM and found that these lncRNAs had a lower expression level than mRNAs (Figure 1b), and $13.7 \%$ of them were specifically detected in cold or normal conditions. We also found that the expression levels of the sense and intronic lncRNAs were higher than those of other types but slightly lower than mRNAs. By comparing global expression levels of lncRNAs between normal and cold-treated conditions (Figure 1c), we detected 316 lncRNAs that were significantly altered by cold treatment, of which 139 showed induced expression (Figure 1d-e, Table S1).

\subsection{The expression pattern and subcellular localization of CRIR1}

Induction of lncRNAs after cold treatment suggested a role of these lncRNAs in cold stress response. In order to test this hypothesis, we selected a transcript of $329 \mathrm{bp}$ and named it COLD-RESPONSIVE IincRNA 1 (CRIR1) for further study. CRIR1 has a short reading frame with 42 amino acids, but it did not 
encode a protein, showing no homology to any parts of any known proteins. The CRIR1gene was located in chromosome 08 between Manes.08G068600 and Manes.08G068500, and there was no overlap between the CRIR1 and its neighbor genes. Like many lncRNAs, the sequence of cassavaCRIR1 showed no clear homology to other genes and had no homologs in other different plants species. Data from the RNA-seq indicated that CRIR1 is expressed at a low level in the plant shoot apex. In order to further examine the spatial expression pattern of $C R I R 1$, total RNA was extracted from different tissues of the wild-type cassava seedlings. The expression level of CRIR1 was determined using qRT-PCR. CRIR1 were preferentially expressed in plant shoots rather than in roots and showed a higher abundance in young leaves (Figure 2a). We further investigated the regulation of CRIR1 by cold stress. Consistent with our RNA-seq data, the CRIR1transcript abundance increased by about 20-fold in cold-stressed seedlings compared with the control seedlings, confirming our result that cold stress strongly induces the expression of CRIR1 (Figure $2 \mathrm{~b}$ ). To investigate the subcellular localization of CRIR1 transcripts, fluorescence in situ hybridization (FISH) in leaves and roots was performed on CRIR1 with a Cy3-labeled probe. Fluorescence signal could be seen in the nucleus and cytoplasm of leaf or root cells hybridized with the Cy3-labeled CRIR1 probe (Figure 2c). These data suggest that CRIR1 transcripts are mainly localized in nucleus and cytoplasm.

\subsection{CRIR1 plays a positive role in plant response to cold stress}

To investigate the physiological function of CRIR1 in planta, we conducted genetic transformation experiments forgenerating transgenic cassava lines with knock-down or overexpression of CRIR1 . Full-length sequences of antisense or sense CRIR1 were transformed into cassava friable embryogenic calli under the control of the 35S Cauliflower Mosaic Virus (CaMV) promoter (35S ::anti-CRIR1 and 35S ::CRIR1). However, the calli carrying the $35 S::$ anti-CRIR1 construct failed to regenerate into complete plantlets, so the basis for this phenomenon still needs to be investigated. Luckily, eight independent transgenic cassava lines carrying the $35 S:: C R I R 1$ construct were obtained and numbered from \#1 to \#8. The CRIR1 mRNA abundance in these transgenic lines was determined by qRT-PCR analysis. Six independent lines that showed higher expression levels of CRIR1 were obtained: \#1-3, \#5, \#7, and \#8 (Figure 3a). Then, the Southern blotting technique was applied to verify the integration events of $35 S:: C R I R 1$ T-DNA in these lines. Two lines, \#1 and \#5, were detected as single-copy insertion into the genome and therefore selected for further investigation (Figure 3b).

In order to investigate whether overexpression of CRIR 1 confers cold stress tolerance, five-week-old wildtype (WT) and CRIR1 overexpression (OE) seedlings were incubated in growth chambers at $4^{\circ} \mathrm{C}$ for 2 days and then transferred to $26^{\circ} \mathrm{C}$ for 7 days. After the treatment, the WT plants showed withered, dry leaves and could not be recovered till death, whereas the OE seedlings were only slightly affected, with fewer leaves wilting and showed a relatively green and healthy appearance (Figure 3c). Proline, acting as a physico-chemical indicator during cold exposure, has been demonstrated to play an important role in protecting cellular enzymes from denaturation (Li, Yang, Iqbal, Qadri, Shi, Wang, Wu, Fan \& Wu, 2019). Cold stress enhanced the accumulation of proline in the WT and OE lines, but the contents of proline in the transgenic lines were significantly higher than that in the wild type (Figure 3d). Malondialdehyde (MDA), which was considered to be an important indicator of cell membrane system injuries and cellular metabolism deterioration, has also been measured as well. Compared with WT, the MDA contents were consistently lower in OE lines (Figure 3e). In addition to morphological and physiological changes, a clear impact on the ultrastructure of chloroplasts membranes was also observed in leaves of cold-treated cassava plants to further assess the degree of cold stress-induced membrane injury (Figure 3f). Before cold treatment, the chloroplasts of leaf mesophyll cells had normal shapes with the typical arrangement of grana and stroma thylakoids and showed no significant differences between the WT and OE line. After cold treatment, the membrane system of chloroplasts in the WT plants showed disintegration with less organized thylakoids, indicating that chloroplasts were destroyed. In contrast, there were no significant alterations in the OE line, except for the appearance of the starch granules in chloroplasts (Figure 3f). These results indicate that CRIR1 plays a positive role in the response of cassava to cold stress through altering the accumulation of protectant metabolites and preventing the membrane system from cell damage under cold stress. 


\subsection{CRIR1 regulates the expression of genes involved in the stress response}

In order to elucidate the molecular mechanism of CRIR1 -mediated cold resistance, we performed RNAsequencing (RNA-seq) on WT and two OE lines grown under normal (N) and cold-treated (C) conditions. Among 33975 detected genes, 6 129, 5 108, and 6440 differentially expressed genes (DEGs) were identified after cold treatment in wild-type, OE \#1 and OE \#5 plants, respectively, indicating that low temperature induced global changes of gene expressions (Figure 4a, Table S2). To identify DEGs specifically regulated by CRIR1, we compared the transcriptomes between OE lines and WT plants under normal and coldtreated conditions. As shown in the venn diagram (Figure 4b), a total of 285 CRIR1 -responsive DEGs were identified both in the OE\#1 and OE\#5 plants under normal conditions. Among them, $30 \%$ of DEGs were cold-regulated genes. After cold treatment, there were 316 genes differentially regulated by CRIR1, and a strong overlap between the cold-related genes and CRIR1 -responsive DEGs were observed. Subsequently, GO enrichment analysis indicated that these DEGs encode proteins were significantly enriched in biological processes associated with stimulus responses, abiotic stimulus responses, and hormone responses under normal conditions. In cold conditions, a high proportion of DEGs can be classified as monocarboxylic acid metabolic, single-organism metabolic, and secondary metabolite biosynthetic process GO categories (Figure 4c). Furthermore, KEGG pathway analysis showed their functions were significantly enriched in the carotenoid biosynthesis pathway, the starch and sucrose metabolism pathway, and the biosynthesis of secondary metabolites pathway under both normal and cold-treated conditions (Figure S1a).

\subsection{CRIR1 regulates cold stress-related genes in a $C B F$-independent pathway}

We next deployed the genes falling into the category of response to the abiotic stimulus, which included two transcription factors (MeNFYA and MeNAC), GALACTINOL SYNTHASE(MeGOLS ), and ABSCISIC STRESS-RIPENING PROTEIN (MeASR ). We verified the expression level of these genes in OE lines with qRT-PCR (Figure 5a). Consistently, transcription levels of MeNFYA and MeNAC were increased in OE lines compared to WT plants under both normal and cold-treated conditions. Quantification of the MeGOLS showed similar trends, whereas we could only detectMeASR after cold stress, suggesting that MeASR is accumulated under low temperature more than under normal conditions. In our RNA-seq analysis, we also found that several IAA-signaling genes were expressed at a lower level in cold-treated OE seedlings than in WT seedlings. These genes include transcription factor YABBY2, AUXIN-RESPONSIVE PROTEIN (IAA18), and AUXIN-BINDING PROTEIN (ABP19). Real-time PCR analysis showed a consistent result with transcriptomic data (Figure 5b). Additionally, we examined the expression patterns of classical cold stress markers, including MeCBFs, MeCORs, and MeICE1. Accumulated evidence has revealed that CBF proteins function as key regulators of the transcriptomic rearrangement leading to cold acclimation. In this study, the WT seedlings showed increased MeCBFs and MeCOR expression levels under cold stress. However, the expression levels of these genes induced by cold stress in the OE seedlings were similar to those of the wild type (Figure 5c). In conclusion, these results indicate that modulation of CRIR1 significantly regulates cold stress-related genes in a $C B F$-independent pathway.

\subsection{CRIR1 interacts with MeCSP5 protein}

In order to further investigate the biological function of CRIR1, we performed in vitro RNA pull-down assays and filtered the protein interactome of CRIR1 using mass spectrometry (Figure 6a). A total of 15 proteins, represented by two or more unique peptides, specifically interacted with biotin-labeled sense- CRIR1 but not the control antisense-CRIR1 (Figure 6b). Among them, three proteins, including one member of the Pre-rRNA PROCESSING PROTEIN (RRP5), one 30S ribosomal protein, and one COLD SHOCK DOMAIN CONTAINING PROTEIN (CSP, named as MeCSP5), were found to be associated withCRIR1 RNA function, and the other candidate interactors were mainly secondary metabolism-related enzymes. CSP has been shown to regulate RNA processing and function by participating in multiple complexes to confer cold tolerance in Arabidopsis (Sasaki \& Imai, 2011). The cassava genome contains six CSPs (MeCSP1 to 6 ) (Figure S2a-b), two of which (MeCSP1 and MeCSP5 ) were regulated by cold stress according to the RNA-seq data. Therefore, we verified possible direct interactions between CRIR1 and MeCSP5. First, we predicted regions of $C R I R 1$ necessary for interaction with MeCSP5 using the catRAPID database, and 
found that MeCSP5 was preferentially bound to the N-terminal of CRIR1 (Figure 6c). Further, to check the specificity of the interaction between CRIR1 and the MeCSP5 protein, full-length CRIR1 were separated into three truncated forms 1-3, and were synthesized by in vitro transcription to evaluate their ability to bind MeCSP5 protein. As presented in Figure 6d, MeCSP5 co-purified with transcripts of segments 1 and 2 but not segment 3, demonstrating a sequence-specific binding activity of MeCSP5. We also checked the interaction of CRIR1 with MeCSP5 using trimolecular fluorescence complementation (TriFC) assay, a modified version of bimolecular fluorescence complementation (BiFC) assay using an MS2 system as described previously (Seo, Sun, Park, Huang, Yeh, Jung \& Chua, 2017). We observed that CRIR1 was strongly associated with MeCSP5 in both the nucleus and cytoplasm, but the negative control RNA was not (Figure 6e). Collectively, these results suggest an association between CSP complexes and CRIR1 -mediated cold stress tolerance. Considering that CSP proteins have been confirmed to possess transcription anti-termination activity and have also been thought to enhance translation at low temperatures by eliminating stabilized RNA secondary structures (Sasaki \& Imai, 2011). We thus compared the mRNA and protein abundance in WT and transgenic plants. According to the RNA-seq data, transcripts in WT and OE lines have a similar mean abundance under normal conditions. However, low temperature strongly reduced cellular mRNA levels in WT or OE lines (Figure 6f). Besides, we found that the mean protein levels produced from OE lines were significantly higher than that in WT plants under both normal and cold-treated conditions based on the mean protein abundance (Figure $6 \mathrm{~g}$ ). These data indicated that CRIR1 may potentially coordinatilate with MeCSP5 may toincrease translational yield for coping with cold stress.

\section{DISCUSSION}

\subsection{A powerful full-length lncRNA resource in cassava was collected}

Cold stress is one of the major environmental factors that limit plant growth, productivity, and geographic distribution. Most tropical plant species are sensitive to cold stress due to the lack of capacity for cold acclimation. Over the past decades, genetic and transcriptomic screens have identified many genes involved in cold stress response. Many researchers tried to enhance the cold tolerance of cassava using the coldresponsive gene and investigated the corresponding mechanism. However, the regulatory pathways that mediate the cold stress responses are far from being understood. The lncRNAs are important regulators of gene expression. The discovery of lncRNAs has filled gaps in cold-responsive regulatory pathways. In this study, many polys (A)+ lncRNAs were identified systematically, and their expression profile under normal or cold-treated conditions was analyzed using Iso-seq and RNA-seq. Many full-length lncRNAs were found for the first time to be specifically expressed during cold treatment. Therefore, the lncRNAs identified in this paper represent a valuable addition to the cassava lncRNA collection, providing a rich resource for the community to investigate the biology of lncRNAs in plants further.

\subsection{CRIR1 is a novel lncRNA regulating plant tolerance to cold stress}

Plant lncRNAs are expected to play important roles in response to environmental conditions, and thousands of lncRNAs have been identified in various plant species (Li, Eichten, Shimizu, Petsch, Yeh, Wu, Chettoor, Givan, Cole, Fowler, Evans, Scanlon, Yu, Schnable, Timmermans, Springer \& Muehlbauer, 2014, Wang, Chung, Liu, Jang, Kean, Xu \& Chua, 2014, Xiao, Yuan, Guo, Hou, Yin, Zhang \& Li, 2015, Xin, Wang, Yao, Song, Hu, Qin, Xie, Peng, Ni \& Sun, 2011, Zhang, Han, Guo, Liu, Zheng, Wu \& Jin, 2014). Besides, there has been a rapid increase in the number of well-studied lncRNAs involved in abiotic stresses response, such asSVALKA (Kindgren, Ard, Ivanov \& Marquardt, 2018), DRIR(Qin et al., 2017), and Cis-NAT ZmNAC48 (Mao et al., 2021). However, for revealing the functions of plant lncRNA in cold stress response is still lagging because most plant lncRNAs are expressed at low levels and are not evolutionarily conserved across species. In the present study, an intergenic lncRNA, CRIR1, was identified, whose expression was significantly induced by cold stress. Compared with WT, overexpression of CRIR1 in cassava could significantly accumulate high leve of proline, thus leading to enhanced survival rates under cold-treated conditions. The cellular morphological characteristics suggested that the expression of CRIR1 can protect the cellular membrane systems from low temperatures. The discovery of CRIR1 provides a new avenue to explore a previously unknown aspect of plant cold stress response. 


\subsection{CRIR1 regulates cold tolerance through $C B F$-independent pathways}

Initially, we assumed that CRIR1 may function as a cis -acting lncRNA involved in the transcriptional regulation of target genes located in the nearby genomic region. Three genes are located close to CRIR1 . RNA-seq and qRT-PCR results of CRIR 1 transgenic plants showed no difference in the transcript levels of neighboring genes (Figure S3). The results suggested that CRIR1 functioned as a trans -acting lncRNA. The candidate target genes of CRIR1 were screened using an RNA-seq data set of CRIR1 OE lines. RNA-seq results demonstrated that the expression of many cold-related genes was increased in OE plants. For example, two transcription factors, MeNAC and MeNFYA, were upregulated in cold-stressed WT plants and CRIR1 overexpressors. NAC and NFYA genes are both involved in abiotic stress responses, and overexpression of $N A C$ confers tolerance of cold, salt, and drought stresses in various plant species (Shao, Wang \& Tang, 2015). Another two genes that are upregulated in the CRIR1 overexpressors are ASR and GOLS, which have been well documented for their positive roles in plant adaption to cold stresses ( $\mathrm{Li}$, Li, Zuo, Li \& Wei, 2018, Shimosaka \& Ozawa, 2016, Taji, Ohsumi, Iuchi, Seki, Kasuga, Kobayashi, Yamaguchi-Shinozaki \& Shinozaki, 2002, Wang, Hu, Feng, Yang, Huang, Xiao, Liu, Yang \& He, 2016). However, the opposite results were obtained in examining the expression of plant growth-related genes, such as MeYAB2 , auxinresponsive protein MeIAA18, and auxin-binding protein MeABP19. These genes showed a decreased expression pattern during cold stress and inCRIR1 -overexpressing plants. These results suggested a possible interaction of CRIR1 with the auxin signaling pathway. The expression levels of cold stress related marker genes, including $M e C B F s$, MeCOR, and MeICE1, showed no difference between WT and overexpression lines, indicating that $C R I R 1$ positively modulated plant cold tolerance through $C B F$-independent pathways.

\subsection{CRIR1 functions related to MeCSP5 in cassava}

MeCSPs are nucleic acid-binding proteins that contain both cold shock domain (CSD) and DNA-binding domains, which have been widely found in bacteria, animals, and plants. CSPs are important during cold shock response. Besides, the functions of CSPs were first described in Escherichia coli (Goldstein, Pollitt \& Inouye, 1990). During cold stress, cell membrane fluidity and enzyme activity decrease, and transcription and translation efficiency is reduced to stabilize nucleic acid secondary structures. CSPs function as RNA chaperones and counteract these harmful effects by destabilizing secondary structures in target RNA at low temperatures, thus facilitating the translation (Keto-Timonen, Hietala, Palonen, Hakakorpi, Lindstrom \& Korkeala, 2016). Arabidopsis AtCSP3 shares an RNA chaperone function with Escherichia coli cold shock proteins and positively regulates freezing tolerance through a non- $C B F$ pathway (Kim et al., 2009). In this study, CRIR1 was showed to be able to interact with MeCSP5, a homology protein of ArabidopsisAtCSP2 with $50 \%$ identity.. In addition, MeCSP5 protein exhibited a similar cellular distribution pattern with CRIR1 transcripts (Figure S2c). Although cold stress dramatically stimulated the MeCSP5 gene expression, its transcript level was not significantly altered in the CRIR1 -overexpressors (Figure S2d), suggesting that CRIR1 could regulate MeCSP5 at protein level. In Arabidopsi s, AtCSP3 protein has been shown to interact with a range of proteins in the nucleolus, nucleoplasm, and cytoplasm, including ribosomal proteins, Gar1 RNA-binding protein, and mRNA decapping protein. The results suggested that AtCSP3 participated in multiple complexes and possibly regulated RNA processing and translation (Kim, Sonoda, Sasaki, Kaminaka \& Imai, 2013). One ribosomal protein was found to interact with CRIR1, indicating the possible involvement of CRIR1 in ribosome biogenesis and protein translation. Therefore, it will be interesting to determine if the complex that forms with CRIR1, MeCSP5 and other proteins regulates cold stress tolerance in cassava by controlling the RNA unwinding activity or facilitating mRNA translation.

Taken together, in this study, a total of 3004 full-length lncRNAs in cassava were identified, and 316 of them were further characterized as cold-related lncRNAs. Functional analysis shows that CRIR1 can confer cold stress tolerance and participate in MeCSP complexes to enhance translation efficiency at low temperatures. The research has provided a valuable source for studying full-length lncRNAs in cassava and their corresponding candidate genes. CRIR1, as a case in the study, will provide applicable genetic resource for breeding cold tolerant crops without yield penalty.

\section{ACKNOWLEDGEMENTS}


This work was supported by the National Key Research and Development Program of China (2019YFD1001105, 2018YFD1000500), the Central Public-interest Scientific Institution Basal Research Fund for Chinese Academy of Tropical Agricultural Sciences (1630052021026), Hainan Provincial Natural Science Foundation of China (320MS097), and the Natural Science Foundation of China (31701484). We are grateful to Dr. Hongwei Guo and Dr. Kang Yan for providing plasmids 6xMS2 and MSCP.

\section{CONFLICTS OF INTEREST}

The authors declare that they have no competing interests.

\section{AUTHOR CONTRIBUTIONS}

S. Li, W. Wang and M. Peng conceived and designed the experiments; S. Li, S. Dong, Z. Li, L. Zou and P. Zhao performed most of the experiments and analyses with helps from X. Guo; S. Li, and Z. Cheng wrote the original draft; X.Guo, P. Zhao, Y. Bao, W. Wang and M. Peng reviewed and edited the manuscript. All authors have read and agreed to the published version of the manuscript.

\section{DATA AVAILABILITY STATEMENT}

The data that supports the findings of this study are available in the supporting materials of this article.

\section{ORCID}

Shuxia Li : https://orcid.org/ 0000-0001-7329-9259

Yan Bao : https://orcid.org/ 0000-0002-9870-5943

Ming Peng : //orcid.org/0000-0003-4843-2773

\section{REFERENCES}

An D., Ma Q., Wang H., Yang J., Zhou W. \& Zhang P. (2017) Cassava C-repeat binding factor 1 gene responds to low temperature and enhances cold tolerance when overexpressed in Arabidopsis and cassava.Plant Mol Biol, 94, 109-124.

An D., Yang J. \& Zhang P. (2012) Transcriptome profiling of low temperature-treated cassava apical shoots showed dynamic responses of tropical plant to cold stress. BMC Genomics , 13 , 64 .

Chinnusamy V., Gong Z. \& Zhu J.K. (2008) Nuclear RNA export and its importance in abiotic stress responses of plants. Curr Top Microbiol Immunol , 326 , 235-255.

Cox J. \& Mann M. (2008) MaxQuant enables high peptide identification rates, individualized p.p.b.-range mass accuracies and proteome-wide protein quantification. Nat Biotechnol, 26 , 1367-1372.

Csorba T., Questa J.I., Sun Q. \& Dean C. (2014) Antisense COOLAIR mediates the coordinated switching of chromatin states at FLC during vernalization. Proc Natl Acad Sci U S A, 111 , 16160-16165.

Ding Y., Shi Y. \& Yang S. (2020) Molecular Regulation of Plant Responses to Environmental Temperatures. Mol Plant , 13 , 544-564.

Ding Z., Tie W., Fu L., Yan Y., Liu G., Yan W., Li Y., Wu C., Zhang J. \& Hu W. (2019) Strand-specific RNAseq based identification and functional prediction of drought-responsive lncRNAs in cassava.BMC Genomics , 20,214 .

E1-Sharkawy M.A. (2004) Cassava biology and physiology. Plant Mol Biol , 56 , 481-501.

Goldstein J., Pollitt N.S. \& Inouye M. (1990) Major cold shock protein of Escherichia coli. Proc Natl Acad Sci U S A , 87, 283-287.

Gordon S.P., Tseng E., Salamov A., Zhang J., Meng X., Zhao Z., Kang D., Underwood J., Grigoriev I.V., Figueroa M., Schilling J.S., Chen F. \& Wang Z. (2015) Widespread Polycistronic Transcripts in Fungi Revealed by Single-Molecule mRNA Sequencing. PLoS One, 10, e0132628. 
Hu W.L., Jin L., Xu A., Wang Y.F., Thorne R.F., Zhang X.D. \& Wu M. (2018) GUARDIN is a p53-responsive long non-coding RNA that is essential for genomic stability. Nat Cell Biol, 20 , 492-502.

Karlson D., Nakaminami K., Toyomasu T. \& Imai R. (2002) A cold-regulated nucleic acid-binding protein of winter wheat shares a domain with bacterial cold shock proteins. J Biol Chem ,277, 35248-35256.

Keto-Timonen R., Hietala N., Palonen E., Hakakorpi A., Lindstrom M. \& Korkeala H. (2016) Cold Shock Proteins: A Minireview with Special Emphasis on Csp-family of Enteropathogenic Yersinia. Front Microbiol $, 7,1151$.

Kim D., Langmead B. \& Salzberg S.L. (2015) HISAT: a fast spliced aligner with low memory requirements. Nat Methods, 12,357-360.

Kim J.S., Park S.J., Kwak K.J., Kim Y.O., Kim J.Y., Song J., Jang B., Jung C.H. \& Kang H. (2007) Cold shock domain proteins and glycine-rich RNA-binding proteins from Arabidopsis thaliana can promote the cold adaptation process in Escherichia coli. Nucleic Acids Res ,35 , 506-516.

Kim M.H., Sasaki K. \& Imai R. (2009) Cold shock domain protein 3 regulates freezing tolerance in Arabidopsis thaliana. J Biol Chem, 284, 23454-23460.

Kim M.H., Sonoda Y., Sasaki K., Kaminaka H. \& Imai R. (2013) Interactome analysis reveals versatile functions of Arabidopsis COLD SHOCK DOMAIN PROTEIN 3 in RNA processing within the nucleus and cytoplasm. Cell Stress Chaperones, 18 , 517-525.

Kindgren P., Ard R., Ivanov M. \& Marquardt S. (2018) Transcriptional read-through of the long non-coding RNA SVALKA governs plant cold acclimation. Nat Commun, 9, 4561.

Lee B.H., Kapoor A., Zhu J. \& Zhu J.K. (2006) STABILIZED1, a stress-upregulated nuclear protein, is required for pre-mRNA splicing, mRNA turnover, and stress tolerance in Arabidopsis. Plant Cell ,18 , 1736-1749.

Li B. \& Dewey C.N. (2011) RSEM: accurate transcript quantification from RNA-Seq data with or without a reference genome. BMC Bioinformatics, 12, 323.

Li H. (2018) Minimap2: pairwise alignment for nucleotide sequences.Bioinformatics , 34 , 3094-3100.

Li J., Xiang C.Y., Yang J., Chen J.P. \& Zhang H.M. (2015) Interaction of HSP20 with a viral RdRp changes its sub-cellular localization and distribution pattern in plants. Sci Rep , 5 , 14016.

Li J., Yang Y., Iqbal A., Qadri R., Shi P., Wang Y., Wu Y., Fan H. \& Wu G. (2019) Correlation analysis of cold-related gene expression with physiological and biochemical indicators under cold stress in oil palm.PLoS One, 14, e0225768.

Li L., Eichten S.R., Shimizu R., Petsch K., Yeh C.T., Wu W., Chettoor A.M., Givan S.A., Cole R.A., Fowler J.E., Evans M.M., Scanlon M.J., Yu J., Schnable P.S., Timmermans M.C., Springer N.M. \& Muehlbauer G.J. (2014) Genome-wide discovery and characterization of maize long non-coding RNAs. Genome Biol, $15, \mathrm{R} 40$.

Li S., Yu X., Cheng Z., Zeng C., Li W., Zhang L. \& Peng M. (2020) Large-scale analysis of the cassava transcriptome reveals the impact of cold stress on alternative splicing. $J$ Exp Bot , $\mathbf{7 1}, 422-434$.

Li S., Yu X., Lei N., Cheng Z., Zhao P., He Y., Wang W. \& Peng M. (2017) Genome-wide identification and functional prediction of cold and/or drought-responsive lncRNAs in cassava. Sci Rep ,7 , 45981.

Li X., Li L., Zuo S., Li J. \& Wei S. (2018) Differentially expressed ZmASR genes associated with chilling tolerance in maize (Zea mays) varieties. Funct Plant Biol , 45 , 1173-1180.

Lindquist J.A. \& Mertens P.R. (2018) Cold shock proteins: from cellular mechanisms to pathophysiology and disease. Cell Commun Signal ,16, 63. 
Liu J., Jung C., Xu J., Wang H., Deng S., Bernad L., Arenas-Huertero C. \& Chua N.H. (2012) Genome-wide analysis uncovers regulation of long intergenic noncoding RNAs in Arabidopsis. Plant Cell ,24, 4333-4345.

Love M.I., Huber W. \& Anders S. (2014) Moderated estimation of fold change and dispersion for RNA-seq data with DESeq2. Genome Biol ,15, 550 .

Malik A.I., Kongsil P., Nguyen V.A., Ou W., Sholihin, Srean P., Sheela M.N., Becerra Lopez-Lavalle L.A., Utsumi Y., Lu C., Kittipadakul P., Nguyen H.H., Ceballos H., Nguyen T.H., Selvaraj Gomez M., Aiemnaka P., Labarta R., Chen S., Amawan S., Sok S., Youabee L., Seki M., Tokunaga H., Wang W., Li K., Nguyen H.A., Nguyen V.D., Ham L.H. \& Ishitani M. (2020) Cassava breeding and agronomy in Asia: 50 years of history and future directions. Breed Sci, 70, 145-166.

Mao Y., Xu J., Wang Q., Li G., Tang X., Liu T., Feng X., Wu F., Li M., Xie W. \& Lu Y. (2021) A natural antisense transcript acts as a negative regulator for the maize drought stress response gene ZmNAC48. $J$ Exp Bot, $\mathbf{7 2}, 2790-2806$.

Melencion S.M.B., Chi Y.H., Pham T.T., Paeng S.K., Wi S.D., Lee C., Ryu S.W., Koo S.S. \& Lee S.Y. (2017) RNA Chaperone Function of a Universal Stress Protein in Arabidopsis Confers Enhanced Cold Stress Tolerance in Plants. Int $J$ Mol Sci, 18 .

Nakaminami K., Karlson D.T. \& Imai R. (2006) Functional conservation of cold shock domains in bacteria and higher plants. Proc Natl Acad Sci U S A , 103, 10122-10127.

Nuruzzaman M., Sharoni A.M. \& Kikuchi S. (2013) Roles of NAC transcription factors in the regulation of biotic and abiotic stress responses in plants. Front Microbiol , 4, 248.

Park S.J., Kwak K.J., Oh T.R., Kim Y.O. \& Kang H. (2009) Cold shock domain proteins affect seed germination and growth of Arabidopsis thaliana under abiotic stress conditions. Plant Cell Physiol ,50 , 869-878.

Pertea M., Pertea G.M., Antonescu C.M., Chang T.C., Mendell J.T. \& Salzberg S.L. (2015) StringTie enables improved reconstruction of a transcriptome from RNA-seq reads. Nat Biotechnol , 33 , 290-295.

Peschansky V.J. \& Wahlestedt C. (2014) Non-coding RNAs as direct and indirect modulators of epigenetic regulation. Epigenetics ,9, 3-12.

Qin T., Zhao H., Cui P., Albesher N. \& Xiong L. (2017) A Nucleus-Localized Long Non-Coding RNA Enhances Drought and Salt Stress Tolerance. Plant Physiol , 175 , 1321-1336.

Rajkowitsch L., Chen D., Stampfl S., Semrad K., Waldsich C., Mayer O., Jantsch M.F., Konrat R., Blasi U. \& Schroeder R. (2007) RNA chaperones, RNA annealers and RNA helicases. RNA Biol , 4 , 118-130.

Rigo R., Bazin J., Romero-Barrios N., Moison M., Lucero L., Christ A., Benhamed M., Blein T., Huguet S., Charon C., Crespi M. \& Ariel F. (2020) The Arabidopsis lncRNA ASCO modulates the transcriptome through interaction with splicing factors. EMBO Rep , 21, e48977.

Sasaki K. \& Imai R. (2011) Pleiotropic roles of cold shock domain proteins in plants. Front Plant Sci , 2 , 116.

Seo J.S., Sun H.X., Park B.S., Huang C.H., Yeh S.D., Jung C. \& Chua N.H. (2017) ELF18-INDUCED LONG-NONCODING RNA Associates with Mediator to Enhance Expression of Innate Immune Response Genes in Arabidopsis.Plant Cell , 29 , 1024-1038.

Shao H., Wang H. \& Tang X. (2015) NAC transcription factors in plant multiple abiotic stress responses: progress and prospects. Front Plant Sci , 6 , 902.

Shi Y., Ding Y. \& Yang S. (2018) Molecular Regulation of CBF Signaling in Cold Acclimation. Trends Plant Sci , 23, 623-637. 
Shimosaka E. \& Ozawa K. (2016) Overexpression of cold-inducible wheat galactinol synthase confers tolerance to chilling stress in transgenic rice. Breed Sci, 65, 363-371.

Song Y., Chen P., Liu P., Bu C. \& Zhang D. (2020) High-Temperature-Responsive Poplar lncRNAs Modulate Target Gene Expression via RNA Interference and Act as RNA Scaffolds to Enhance Heat Tolerance. Int $J$ Mol Sci, 21 .

Taji T., Ohsumi C., Iuchi S., Seki M., Kasuga M., Kobayashi M., Yamaguchi-Shinozaki K. \& Shinozaki K. (2002) Important roles of drought- and cold-inducible genes for galactinol synthase in stress tolerance in Arabidopsis thaliana. Plant $J, 29,417-426$.

Tian T., Liu Y., Yan H., You Q., Yi X., Du Z., Xu W. \& Su Z. (2017) agriGO v2.0: a GO analysis toolkit for the agricultural community, 2017 update. Nucleic Acids Res , 45, W122-W129.

Waititu J.K., Zhang C., Liu J. \& Wang H. (2020) Plant Non-Coding RNAs: Origin, Biogenesis, Mode of Action and Their Roles in Abiotic Stress.Int J Mol Sci, 21 .

Wang H., Chung P.J., Liu J., Jang I.C., Kean M.J., Xu J. \& Chua N.H. (2014) Genome-wide identification of long noncoding natural antisense transcripts and their responses to light in Arabidopsis. Genome Res , $\mathbf{2 4}, 444-453$.

Wang J., Meng X., Dobrovolskaya O.B., Orlov Y.L. \& Chen M. (2017) Non-coding RNAs and Their Roles in Stress Response in Plants. Genomics Proteomics Bioinformatics , 15 , 301-312.

Wang L., Hu W., Feng J., Yang X., Huang Q., Xiao J., Liu Y., Yang G. \& He G. (2016) Identification of the ASR gene family from Brachypodium distachyon and functional characterization of BdASR1 in response to drought stress. Plant Cell Rep , 35, 1221-1234.

Xiao H., Yuan Z., Guo D., Hou B., Yin C., Zhang W. \& Li F. (2015) Genome-wide identification of long noncoding RNA genes and their potential association with fecundity and virulence in rice brown planthopper, Nilaparvata lugens. BMC Genomics, 16 , 749.

Xiao L., Shang X.H., Cao S., Xie X.Y., Zeng W.D., Lu L.Y., Chen S.B. \& Yan H.B. (2019) Comparative physiology and transcriptome analysis allows for identification of lncRNAs imparting tolerance to drought stress in autotetraploid cassava. BMC Genomics , $20,514$.

Xin M., Wang Y., Yao Y., Song N., Hu Z., Qin D., Xie C., Peng H., Ni Z. \& Sun Q. (2011) Identification and characterization of wheat long non-protein coding RNAs responsive to powdery mildew infection and heat stress by using microarray analysis and SBS sequencing. BMC Plant Biol , 11 , 61.

Yang W., Schuster C., Prunet N., Dong Q., Landrein B., Wightman R. \& Meyerowitz E.M. (2019) Visualization of Protein Coding, Long Noncoding, and Nuclear RNAs by Fluorescence in Situ Hybridization in Sections of Shoot Apical Meristems and Developing Flowers. Plant Physiol ,182 , 147-158.

Yang Y. \& Karlson D. (2013) AtCSP1 regulates germination timing promoted by low temperature. FEBS Lett , 587 , 2186-2192.

Zhang P., Potrykus I. \& Puonti-Kaerlas J. (2000) Efficient production of transgenic cassava using negative and positive selection. Transgenic Res , 9 , 405-415.

Zhang W., Han Z., Guo Q., Liu Y., Zheng Y., Wu F. \& Jin W. (2014) Identification of maize long non-coding RNAs responsive to drought stress. PLoS One, 9 , e98958.

Zhao C., Zhang Z., Xie S., Si T., Li Y. \& Zhu J.K. (2016) Mutational Evidence for the Critical Role of CBF Transcription Factors in Cold Acclimation in Arabidopsis. Plant Physiol , 171 , 2744-2759.

Zhao X., Li J., Lian B., Gu H., Li Y. \& Qi Y. (2018) Global identification of Arabidopsis lncRNAs reveals the regulation of MAF4 by a natural antisense RNA. Nat Commun, 9, 5056.

Zhu J.K. (2016) Abiotic Stress Signaling and Responses in Plants. Cell , 167 , 313-324. 


\section{SUPPORTING INFORMATION}

FIGURE S1 KEGG pathway enrichment analysis of CRIR1 -regulated genes under normal and cold-treated conditions, respectively.

FIGURE S2 Phylogenetic and subcellular localization analysis of cassava MeCSPs .

FIGURE S3 Expression analysis of the CRIR1 neighbors.

Table S1 Information of lncRNAs identified from Iso-seq data.

Table S2 Information of the DEGs in different comparison groups.

Table S3 List of primers used in this study.

Figure legends

FIGURE 1 Identification of cold-responsive lncRNAs in cassava. (a) The type and number of predicted lncRNAs. Intergenic lncRNA: lncRNA transcribed from a position

between two annotated protein-coding genes and are at least $1 \mathrm{~kb}$ away from the nearest protein-coding genes. Bidirectional LncRNA: lncRNA is oriented head-to-head with a protein-coding gene within $1 \mathrm{~kb}$. Intronic lncRNA: lncRNA overlaps with the intron of annotated coding genes in either sense or antisense orientation. Antisense lncRNA: lncRNA has opposite transcriptional direction and overlap in part with adjacent mRNA. Sense lncRNA: lncRNA has the same transcriptional direction and overlaps with adjacent mRNA. (b) Expression profiles of lncRNA and mRNA under normal conditions. FPKM, fragments per kilobase of exons per million fragments mapped. (c) The abundance of all the expressed lncRNAs in each sample. (d) Volcano plot of differentially expressed genes between normal and cold-treated conditions. (e) The number of differentially expressed lncRNAs in response to cold stress. The red bar represents upregulated lncRNAs, and the blue bar represents down-regulated lncRNAs.

FIGURE 2 The expression pattern and subcellular distribution of CRIR1 in cassava. (a) Spatial expression pattern analysis of CRIR1 in different tissues using quantitative RT-PCR. (b) Expression levels of CRIR1 in cassava leaves responding to cold stress. Cassava plants without stress were used as the controls to normalize the expression in the stress treatments. Error bars indicate the sd of three biological replicates. (c) Subcellular distribution of CRIR1 in cassava leaf cells. CRIR1 transcripts were detected by in situ hybridizations of Cy3-tagged oligonucleotides (green). U6 (yellow) was used as a control. The location of nuclei is indicated by DAPI staining (blue). Bar $=20 \mu \mathrm{m}{ }^{*} \mathrm{P}<0.05,{ }^{* *} \mathrm{P}<0.01$ by Student's $t$-test.

FIGURE 3 Effects of $C R I R 1$ on plant growth, proline accumulation, and cellular membrane stability during cold stress. (a) Relative transcript levels of CRIR1 in the wild-type (WT) and eight overexpressing lines, \#1 to \#8. (b) Transgene integration pattern in WT and OE lines by Southern analysis. (c) Morphology of pot-grown seedlings before and after cold stress treatment $(4,48 \mathrm{~h}$ ) for one-week recovery under normal growth conditions. Bar $=2 \mathrm{~cm}$. (d, e) Proline concentration (d) and MDA level (e) in the WT and transgenic plants under cold treatment (4) for 0 and $24 \mathrm{~h}$. FW, fresh weight. (f) Transmission electron microscopic (TEM) analysis of chloroplasts from WT and OE \#1 young leaves under normal and cold-treated conditions (4, 48 h). cp, chloroplast. Bar $=2 \mu \mathrm{m}$. Data represent means $\pm \mathrm{SD},{ }^{*} \mathrm{P}<0.05,{ }^{*} \mathrm{P}<0.01$ by Student's $t$-test.

FIGURE 4 Identification of CRIR 1 -regulated genes and their corresponding signaling pathways. (a) The numbers of differentially expressed genes (DEGs) across seven comparisons. The red bar represents upregulated DEGs, and the blue bar represents down-regulated DEGs. (b) Venn diagrams show the numbers of the CRIR1 -regulated genes between two OE lines, overlapping and non-overlapping parts with cold-response genes under normal and cold-treated conditions, respectively. (c) Gene Ontology (GO) analysis of CRIR1 -regulated genes under normal and cold-treated conditions, respectively. The top 10 biological processes GO terms are shown.

FIGURE 5 Relative expression levels of selected genes in cold-treated seedlings. (a-c) Relative expression levels of CRIR1 -regulated genes $(\mathrm{a}, \mathrm{b})$ and cold-responsive marker genes (c) are detected by qRT-PCR. Data 
represent means $\pm \mathrm{SD},{ }^{*} \mathrm{P}<0.05,{ }^{*} \mathrm{P}<0.01$ by Student's $t$-test.

FIGURE 6 CRIR1 interacts with MeCSP5 in cassava and improves translational yield. (a) Silver staining and mass spectrometry analyses following RNA pull-down of CRIR1 sense or CRIR1 antisense (AS-CRIR1) in cassava leaf cells. (b) Fifteen candidates for CRIR1 -interacted proteins are identified by mass spectrometry analyses. MW, molecular weight. (c) The RNA binding site prediction of CRIR1- MeCSP5 complex using catRAPID. (d) Confirmation of binding of MeCSP5 to CRIR1 with EMSA. NC, negative control. (e) TriFC assay in rice protoplasts. nYFP is fused to MeCSP5, and cYFP is fused to MSCP. 6xMS2 nucleotide sequences are fused to CRIR1 or control lncRNA. Bars $=10 \mu \mathrm{m}$. (f) The abundances of mRNAs in WT and transgenic lines under normal and cold-treated conditions are shown. The Density (y-axis) corresponds to the relative number of genes at a given abundance. (g) Total protein abundances in WT and transgenic lines under normal and cold-treated conditions are shown. Data represent means $\pm \mathrm{SD},{ }^{*} \mathrm{P}<0.05$ by Student's $t$-test.

(a)

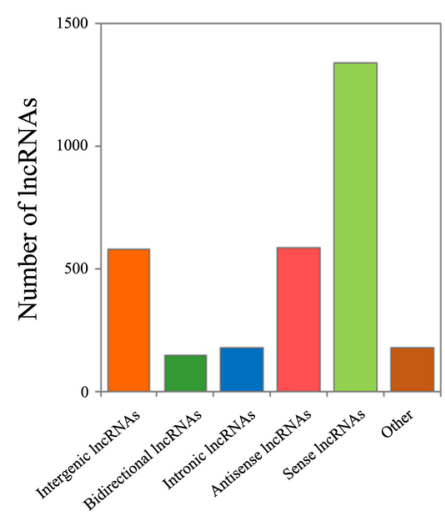

(c)

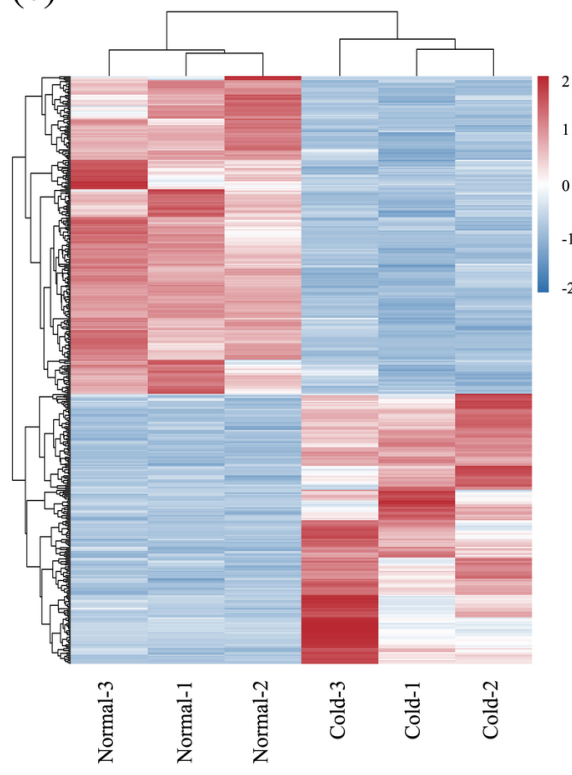

(b)

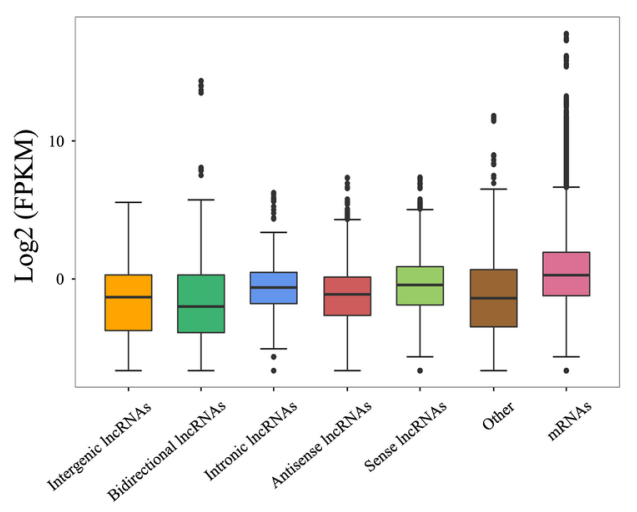

(d)
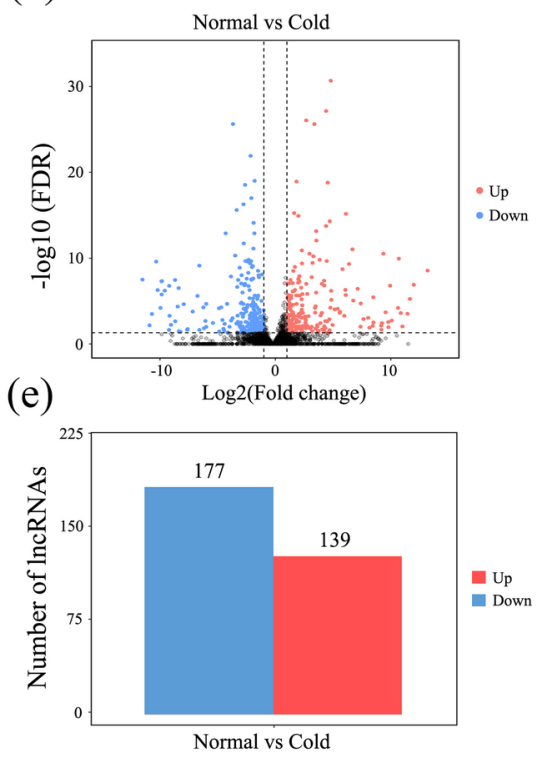
(a)

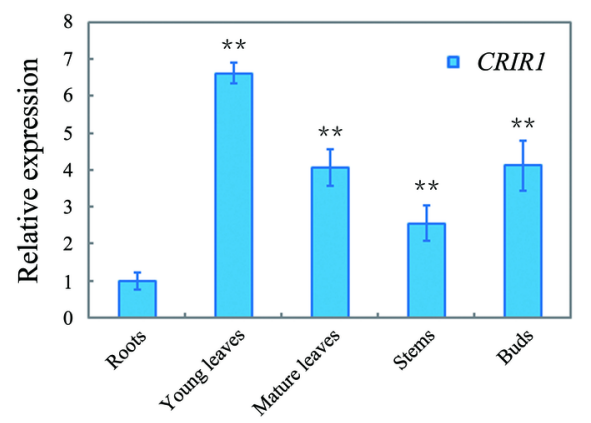

(b)

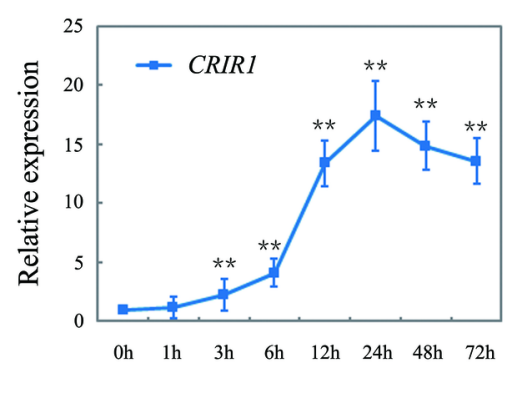

(c)

DAPI

Labeled CRIR 1 probe $\quad$ Labeled $U 6$ probe

Merge
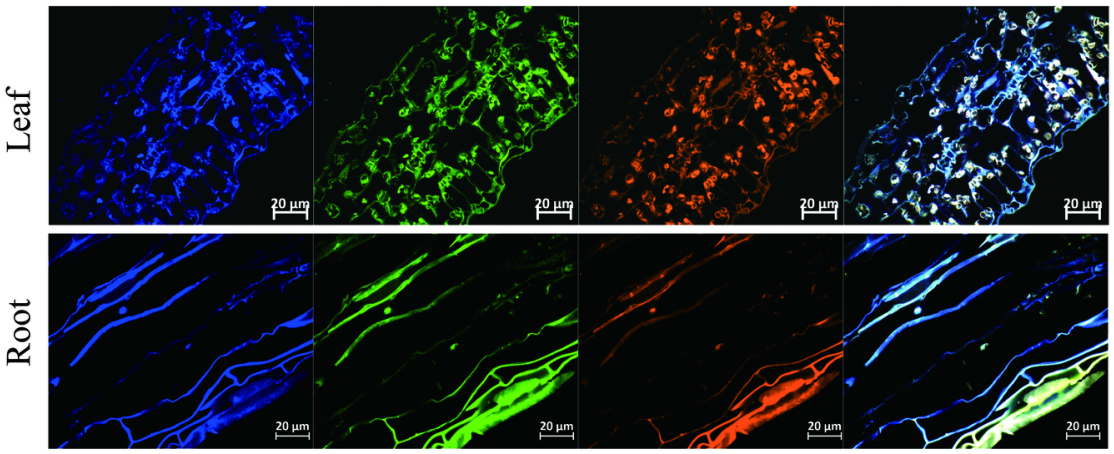
(a)

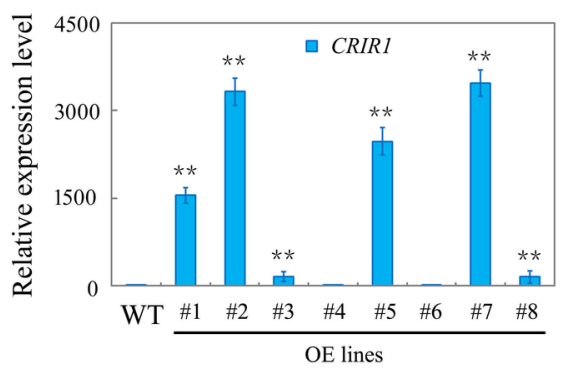

(c)

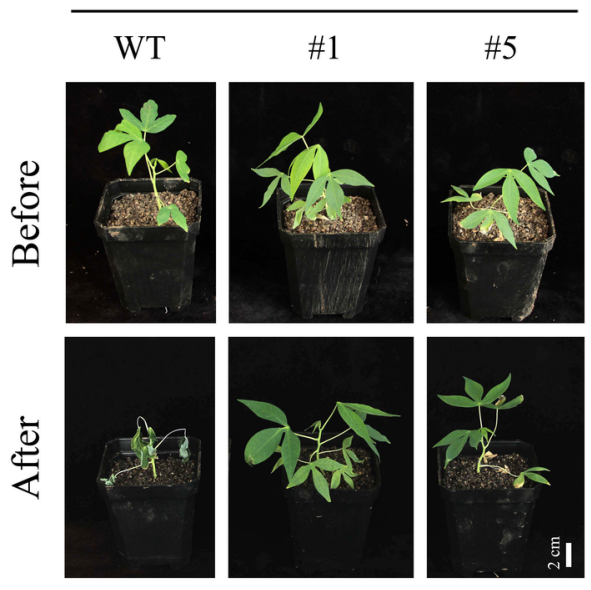

(f)

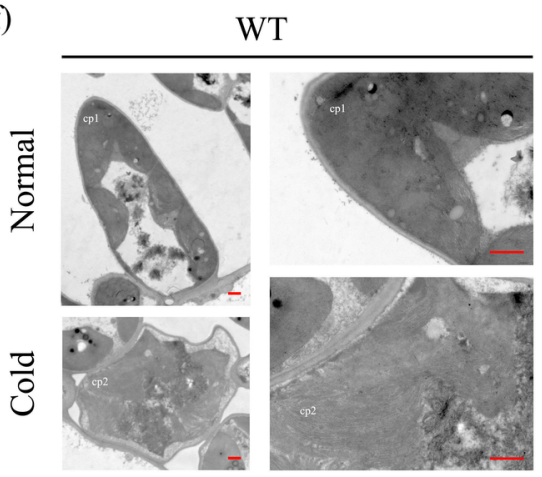

(b) OE lines

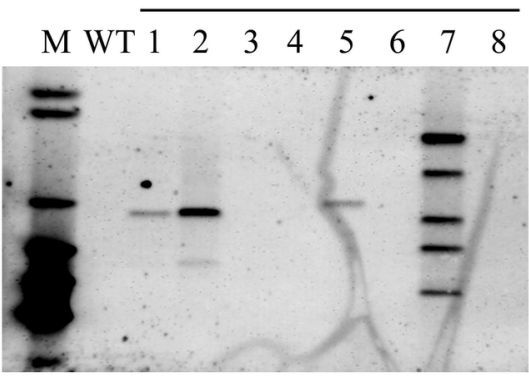

(d)

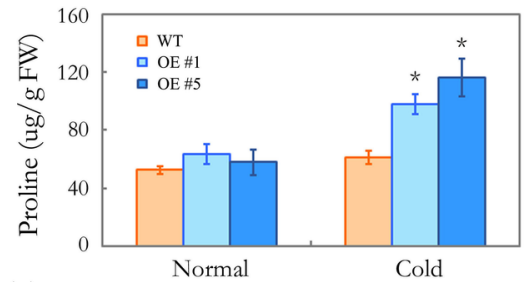

(e)

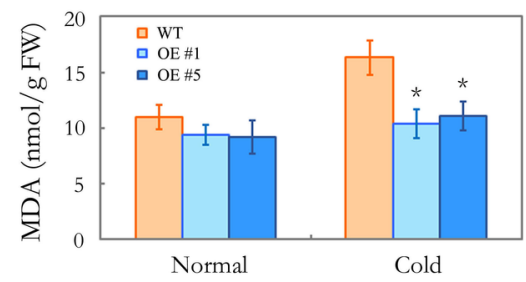

CRIR1 OE \#1

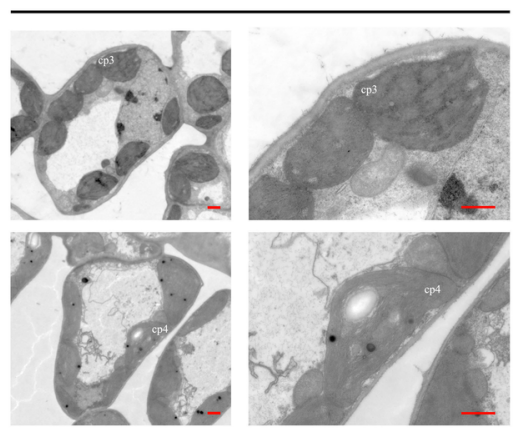


(a)

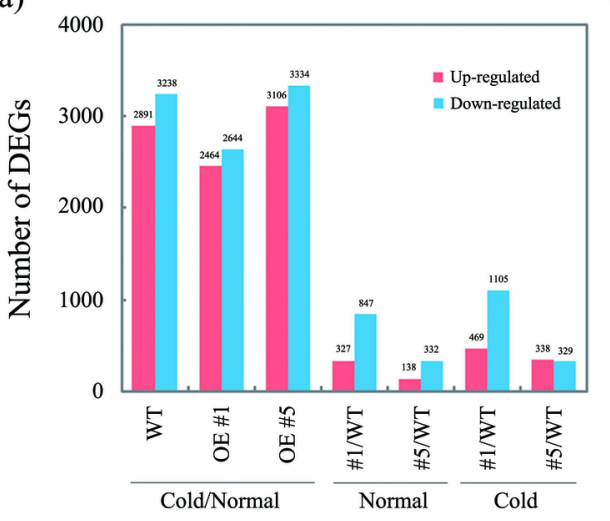

(b)

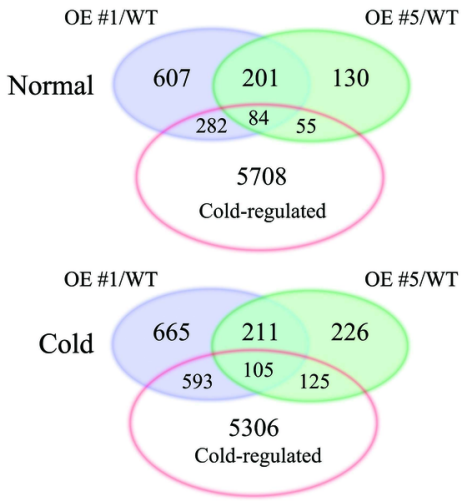

(c)
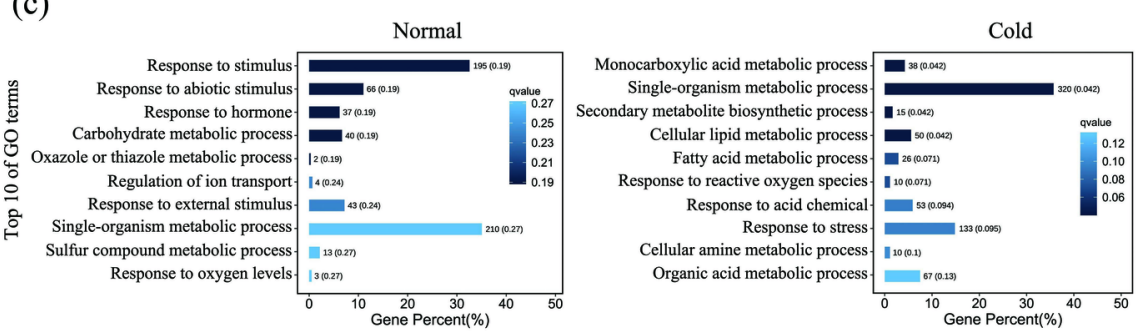

(a)
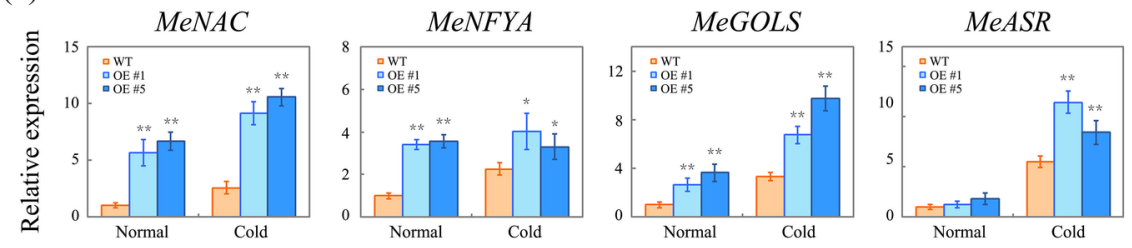

(b)
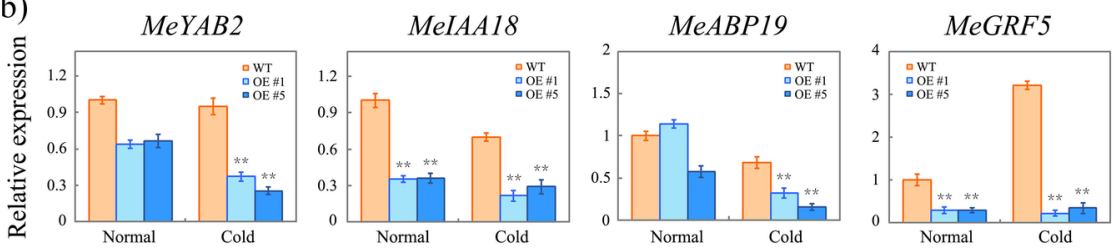

(c)
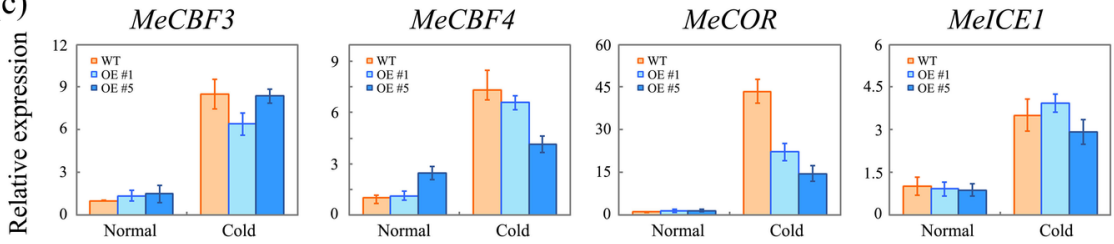
(a)

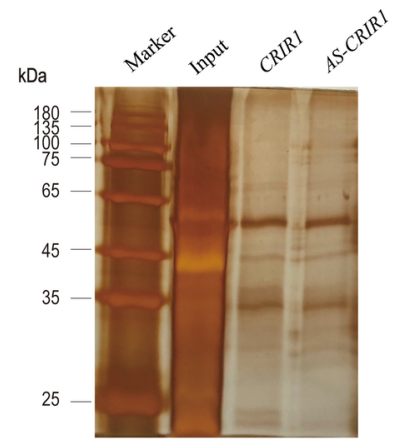

(c)

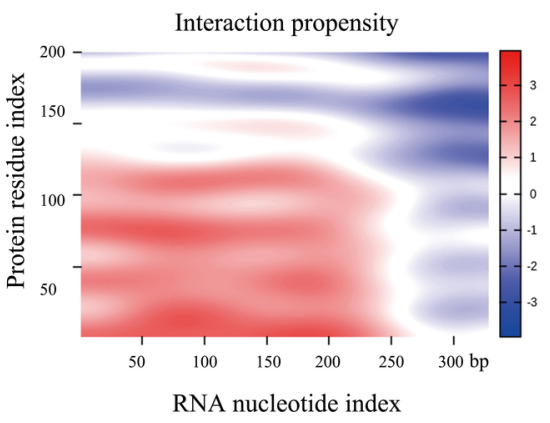

(b)

\begin{tabular}{|c|c|c|c|}
\hline Gene ID & Name & Coverage[\%] & $\mathrm{MW}[\mathrm{kDa}]$ \\
\hline Manes.08G139400 & Jumonji domain-containing protein & 0.9 & 142.35 \\
\hline Manes.5006300 & PPR repeat family & 1.3 & 112.48 \\
\hline Manes.09G127900 & U3 small nucleolar RNA-associated protein 13 & 1.2 & 98.369 \\
\hline Manes. .052700 & PPR repeat family & 1.4 & 82.38 \\
\hline Manes. $09 \mathrm{G} 084000$ & Ribosomal protein 86 kinase & 2.1 & 67.398 \\
\hline Manes. $17 \mathrm{G} 081200$ & GPI mannosyltransferase 2 & 4.3 & 57.294 \\
\hline Mancs. $15 \mathrm{G} 048700$ & Pectatc lyase 1-related & 7.8 & 49.344 \\
\hline Manes. $05 \mathrm{G} 193800$ & Pre-rRNA processing protein RRP5 & 2.6 & 43.119 \\
\hline Manes. $09 \mathrm{G} 171600$ & Serine threonine protein phosphatase & 3.7 & 36.196 \\
\hline Manes. $08 \mathrm{G} 099000$ & Xyloglucan endotransglycosylase & 3.8 & 34.103 \\
\hline Manes. $.7 \mathrm{G} 068000$ & Kynurenine formamidase & 12 & 30.689 \\
\hline Manes. $18 \mathrm{G} 074700$ & Cold shock domain-containing protein & 4.5 & 20.184 \\
\hline Manes. $04 \mathrm{G} 130000$ & 30 S ribosomal protein S17 & 6.3 & 17.814 \\
\hline Manes. $03 \mathrm{G} 115200$ & DM DNA-binding domain-contain & 16.2 & 7.924 \\
\hline Mancs.13G080500 & Hypothetical protein & 27.3 & 5.028 \\
\hline
\end{tabular}

(d)

CRIRI fragments
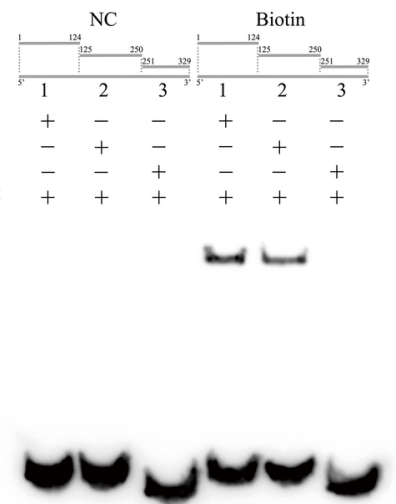

(g)

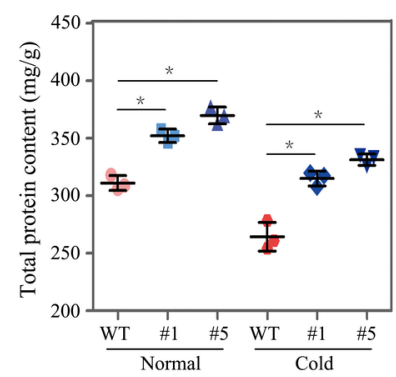

